\title{
Optimization of Finishing Parameters for Magnetic Compound Fluid Finishing (MCFF) of Copper Alloy
}

Jinwei Fan ( $\nabla$ jwfan@bjut.edu.cn )

Beijing University of Technology https://orcid.org/0000-0002-7766-9234

\section{Xingfei Ren}

Beijing University of Technology

\section{Ri Pan}

Beijing University of Technology

\section{Peitong Wang}

Beijing University of Technology

Haohao Tao

Beijing University of Technology

\section{Research Article}

Keywords: Magnetic compound fluid finishing, Neural network, Dimensional accuracy, Finishing parameters, Surface roughness

Posted Date: October 20th, 2021

DOl: https://doi.org/10.21203/rs.3.rs-989241/v1

License: (c) (1) This work is licensed under a Creative Commons Attribution 4.0 International License.

Read Full License 


\title{
Optimization of finishing parameters for magnetic compound fluid finishing (MCFF) of
}

\section{copper alloy}

Jinwei Fan ${ }^{1 *}$, Xingfei Ren ${ }^{1}$, Ri Pan ${ }^{1}$, Peitong Wang ${ }^{1}$, Haohao Tao ${ }^{1}$

${ }^{1}$ Faculty of Materials and Manufacturing, Beijing University of Technology, Beijing, 100124, China

${ }^{*}$ Correspondence: Jinwei Fan, Faculty of Materials and Manufacturing, Beijing University of Technology, Beijing, 100124, China.

Email: jwfan@bjut.edu.cn

\begin{abstract}
As an advanced finishing technology, magnetic compound fluid finishing (MCFF) is considered to be able to achieve damagefree finishing of low-hardness materials such as copper alloys with appropriate finishing parameters. However, ignoring the influence of the material removal amount on the dimensional accuracy when optimizing the finishing parameters may result in excessive material removal and a reduction in the workpiece's dimensional accuracy. Thus, a novel finishing parameters optimization method considering dimensional accuracy is proposed in this paper. Firstly, the MCFF experiments are planned and carried out for modeling. Secondly, a MCFF model is established based on the integrated learning theory. The established model is a multi-layer neural network fusion model comprised of a prediction layer and a fusion layer, which can accurately predict the polished surface quality and material removal amount. Thirdly, taking the effect of material removal amount on the dimensional accuracy into account, the finishing parameters are optimized by the multi-objective particle swarm optimization algorithm. Finally, the model's prediction accuracy and the superiority of the optimized parameters are compared and verified by experiments. The results demonstrate that the developed model can correctly predict the finishing effect, and the high-quality polished surfaces and high dimensional accuracy can be obtained with the optimized finishing parameters.
\end{abstract}

Keywords: Magnetic compound fluid finishing, Neural network, Dimensional accuracy, Finishing parameters, Surface roughness

\section{Introduction}

Copper and copper alloys with high-quality surfaces are widely used in mems, integrated circuits, lasers, aerospace, and other industries due to their advantages of high thermal, electrical conductivity, high reflectivity, and lightweight [1]. Therefore, the finishing of copper and copper alloys has been attracting a lot of attention from researchers. Although conventional mechanical finishing, electrochemical polishing (ECP), and chemo-mechanical polishing (CMP) can be used for finishing copper alloys, there are several disadvantages associated with these processes. Abrasive particles will leave scratches on the surface of copper alloys owing to the high normal force of conventional mechanical finishing, resulting in a poor polished surface [2]. And harmful gases are generated during the CMP process [3]. In order to overcome the disadvantages of conventional finishing technologies, many advanced finishing technologies have been applied in the finishing of copper and copper alloys. Among various finishing technologies, magnetic field-assisted finishing (MFAF) is considered to be the most advantageous technology for obtaining a highquality copper surface without damage [2].

MFAF is a type of superfinishing process, which includes abrasive flow finishing (AFF), magnetic float finishing (MFF), magnetorheological finishing (MRF), magnetic compound fluid finishing (MCFF), ball end magnetorheological finishing (BEMRF), and magnetic abrasive finishing (MAF) [4-7]. And MFAF is regarded as the optimal method for achieving high-quality finishing without damage due to the tiny controlled finishing force $\left(1 \times 10^{-4} \mathrm{mN}\right)[6,8]$. Additionally, the material removal amount $(\mathrm{MRA})$ 
caused by MFAF is less than other finishing technologies, which can significantly reduce the effect of finishing on dimensional accuracy. It is worth emphasizing that the magnetic grinding brush formed during MFAF is readily deformed and conforms to the contour of the workpiece surface, making it suitable for finishing 3D free-form surfaces [9]. However, numerous investigations have shown that the finishing effect of MFAF is highly reliant on the preparation of finishing liquid and the selection of finishing parameters. To improve the quality of polished surface, many academics have done extensive studies on the preparation of finishing liquid, the optimization and selection of finishing parameters.

For the preparation of finishing liquid, Sidpara et al. studied the effect of the concentration of CIPs and abrasives on the final surface roughness $(\mathrm{Ra})$ and material removal rate (MRR). The results indicate that Ra decreases and MRR increases with the increase in CIPs concentration, and Ra increases and MRR decreases with the increase in abrasive particles concentration. Finally, the minimized Ra and maximized MRR were obtained with optimized parameters [10]. Niranjan et al. investigated the impact of each component's volume fraction in the finishing liquid on the finishing effect. The optimal finishing liquid ratio was determined via comparison and optimization, and the workpiece's surface roughness was decreased by almost 80\% [11]. Guo et al. compared the finishing performance of larger-sized alumina and smaller-sized zirconia, and pointed out that superior quality can be obtained with smaller-sized zirconia [12]. Li et al. discovered that the abrasive particle size has the most significant influence on the finishing effect, followed by iron powder and abrasive concentrations [2].

Numerous investigations have revealed that the type of carrier medium, the type of abrasive particles, the proportion and size of each component, etc., significantly impact the finishing effect. For various finishing materials and methods, the preparation of the optimal finishing solution varies. Thus, preparing the optimal finishing liquid for the current condition is necessary [13, 14]. Additionally, the magnetic compound fluid (MCF), which is produced by combining magnetic fluid (MF) and magnetorheological fluid (MRF), exhibits superior finishing performance than MF or MRF [15]. Therefore, finishing copper alloys with MCF is a great option.

Appropriate finishing parameters are necessary for producing high-quality polished surfaces. Therefore, further research is being conducted on the optimization and selection of finishing parameters. Guo et al. demonstrated the rotational speed of the magnet has relatively little effect on the finishing process, while the MCF carrier rotational speed and machining gap significantly affect the finishing process [16]. Alam et al. analyzed the influence of magnetic field intensity on the effect of MRF and pointed out that a high magnetic field promotes faster material removal. Additionally, a high-precision surface roughness prediction model was developed based on theoretical research [7]. Based on fluid mechanics and Preston Equation, the influences of various finishing parameters (including magnetic poles, rotational speeds of workpieces, finishing disk, and the machining gap) on finishing pressure were investigated by Pan et al. Furthermore, a MRR model based on multiple regression was established. After 5 hours of finishing with the optimum finishing parameters, the surface roughness of the single-crystal silicon was decreased from $0.48 \mu \mathrm{m}$ to $2.4 \mathrm{~nm}$ [17]. Sidpara et al. developed a prediction model for MRR and Ra using polynomial fitting. Four independent parameters were considered in the modeling: the volume percentage of CIPs and abrasive particles, the carrier wheel rotational speed, and the initial surface roughness. By optimizing the finishing parameters, an excellent finishing effect was obtained [10]. To obtain appropriate finishing parameters, Jiao et al. studied the effects of machining gap and rotational speed on the finishing effect of optical glass. According to research, finishing with a smaller machining gap and higher rotational speed results in a superior surface and a higher MRR [6]. Mosavat et al. modeled and simulated the MAF process with Smoothed Particle Hydrodynamics and analyzed the effects of abrasive particle size, machining gap and rotational speed on roughness and material removal. A damage-less surface with high quality was obtained with the appropriate finishing parameters [18]. Fan et al. developed a magnetic field-assisted finishing tool for titanium alloy finishing, and built an experimental platform to investigate the impact of finishing parameters on finishing quality. The research results indicate that the final finishing quality is determined by the abrasive grain size, the machining gap, the feed rate, and the spindle speed. The titanium alloy surface roughness was reduced from $1.121 \mu \mathrm{m}$ to $46 \mathrm{~nm}$ with the optimized finishing parameters [19].

The majority of the research discussed above has focused on silicon wafers, glass, titanium alloys, and other high-hardness materials, whereas research on the finishing of low-hardness materials is relatively scarce. In fact, MFAF is also capable of achieving 
high-quality finishing on low-hardness materials. In recent years, some scholars have carried out research on obtaining high-quality copper surfaces by MFAF. Prateek et al. studied the process of ultrasonic-assisted MRF of copper alloy and discussed the influence of voltage on the electromagnet, rotational speed, mesh size, and pulse on time of ultrasonic vibration on the surface roughness [20, 21]. Khan et al. conducted an in-depth study on the preparation of the finishing liquid in order to achieve high-quality finishing of copper alloys using BEMRF. Each component's impact on the finishing effect was investigated, and the optimal finishing liquid was produced. The surface roughness of the brass alloy was reduced to $38 \mathrm{~nm}$ by using the optimized finishing liquid [2, 22]. More et al. investigated the effect of finishing parameters on the surface roughness during magnetorheological finishing of copper alloys and developed a surface roughness prediction model. Surface roughness was reduced to $0.033 \mu \mathrm{m}$ from the initial roughness of $0.33 \mu \mathrm{m}$ after finishing for six hours [23].

While extensive research on MFAF has been conducted, the effect of finishing parameters on dimensional accuracy is seldom addressed during the process of optimizing finishing parameters, which may result in excessive material removal and a reduction in dimensional accuracy. For instance, Pan et al. did not consider the effect of material removal on the dimensional accuracy while optimizing the finishing parameters of single-crystal silicon. Although super-smooth uniform surfaces with a roughness of $2.4 \mathrm{~nm}$ were acquired, the sample thickness was decreased by nearly $39 \mu \mathrm{m}$, which would significantly decrease the dimensional accuracy [17].

However, compared to high-hardness materials such as silicon, there is more material removal in the finishing of copper with lower hardness. If the dimensional accuracy is not taken into account while optimizing the finishing parameters for copper, the dimensional accuracy may decrease more obviously. Therefore, taking the influence of the MRA on dimensional accuracy into consideration is necessary.

The objective of this paper is to obtain a workpiece with a high-quality polished surface and high dimensional accuracy through the MCFF technology. This paper is organized as follows: in section II, the MCFF experiment is designed and executed to obtain the finishing data for modeling; in section III, a high-precision multi-neural network fusion model (MNNFM) based on the integrated learning theory is established, which is used to accurately predict the polished surface quality and MRA. In section IV, considering the effect of MRA on the dimensional accuracy, the finishing parameters are optimized by the multi-objective particle swarm optimization (MOPSO) algorithm. Conclusions are drawn in Section V.

\section{Details of the experimental setup}

\subsection{Preparation of $\mathrm{MCF}$}

The MCFF was selected for high-quality copper alloy finishing based on prior research. The MCF is composed of magnetizable particles (Carbonyl Iron Particles (CIPs), etc.), carrier medium (silicone oil, mineral oil, etc.), abrasive particles (diamond abrasives, silicon carbide, aluminium oxide, cerium oxide, etc.), and additives ( $\alpha$-fibre, etc.). According to the research results of Khan et al. [2], the MCF suitable for the copper alloys was prepared through experiments. In the prepared MCF, MRF-250 is used as the carrier liquid, and CIPs are used as magnetic particles because of their spherical form, high permeability, and high purity. The abrasive particles used are cerium oxide, which is the most commonly used material for high-quality finishing. Additionally, a trace of cellulose was added to improve the viscosity of MCF, minimize the settling of solid particles, and produce better quality and efficient finishing. The composition of the prepared MCF is shown in Table 1. It is worth noting that the selected abrasive particles are spherical, not typical abrasive particles with sharp angles. Moreover, the abrasive particles and CIPs are the same in size to provide a superior finishing effect $[10,24]$.

Table 1 Compositions of MCF

\begin{tabular}{ccccc}
\hline Components & MRF-250 & CIPs & Cerium oxide & $\alpha$-cellulose \\
\hline Content & $51 \mathrm{wt} \%$ & $38.7 \mathrm{wt} \%$ & $7 \mathrm{wt} \%$ & $3.3 \mathrm{wt} \%$
\end{tabular}


Fig. 1 a) shows the state of the prepared MCF under the action of a magnetic field. CIPs and cerium oxide are organized in the direction of the lines of magnetic induction by the action of the magnetic field, forming a large number of complex chain structures [25]. These chain structures are collectively called Flexible Magnetic Abrasive Brush (FMAB) [26]. During the finishing process, FMAP rotates with the rotation of the MCF carrier and apply normal force (magnetic pressure and hydrodynamic pressure) and shear force on the surface of the workpiece (as shown in Fig.1 b)). The finishing force can be controlled by adjusting the intensity of the magnetic field and finishing parameters. Material will be removed when the shear force is greater than the force required for material removal, achieving the purpose of finishing.

a)

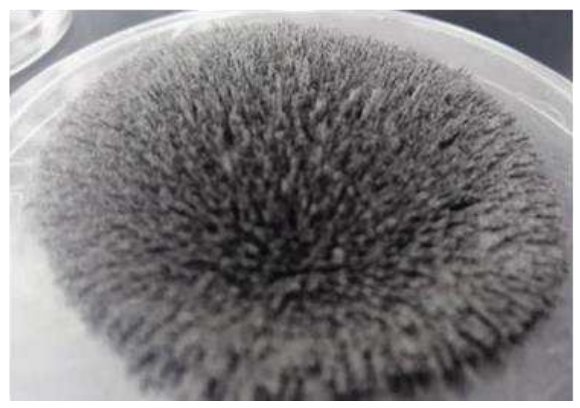

b)

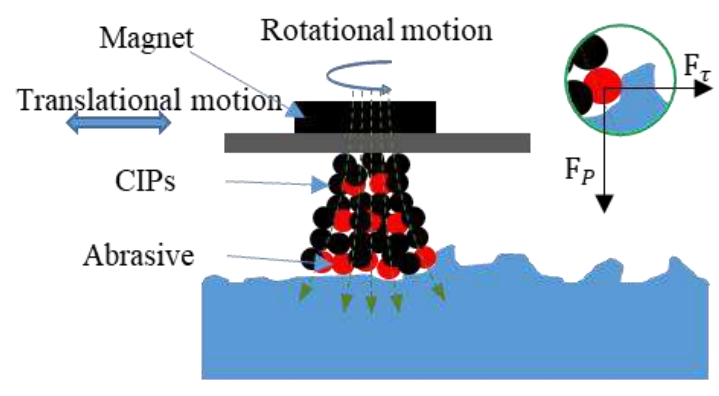

Fig. 1 a) Status of MCF under the action of a magnetic field b) Interaction of MCF and workpiece

\subsection{Experimental platform}

The experiment was carried out on the self-built MCF finishing experimental platform (as shown in Fig. 2 a)). The finishing head has degrees of freedom in the $\mathrm{X}$ and $\mathrm{Z}$ directions, and the processing platform has degrees of freedom in the $\mathrm{Y}$ direction. The finishing head is hollow, with a biased permanent magnet placed within (as shown in Fig. 2 b)). The permanent magnet rotates in the opposite direction to the finishing head throughout the finishing process, forming a dynamic magnetic field. The dynamic magnetic field may assist in regenerating the damaged FMAB and enhancing the efficiency and effect of finishing. The MCF will be adsorbed on the aluminum MCF carrier, which is located at the bottom of the finishing head, under the action of the magnetic field. When the MCF rotates with the finishing head, the surface of the workpiece will be polished by the formed FMAB.

a)

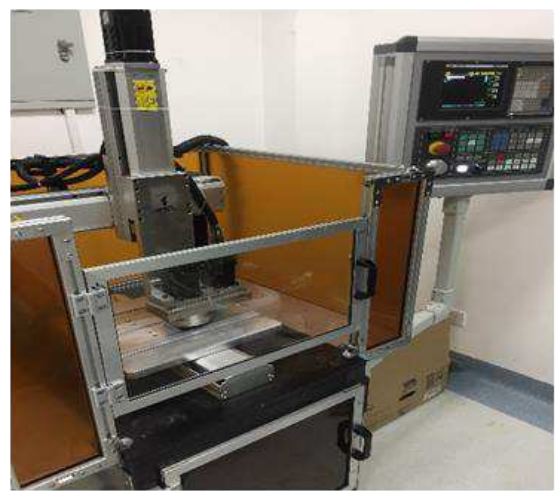

b)

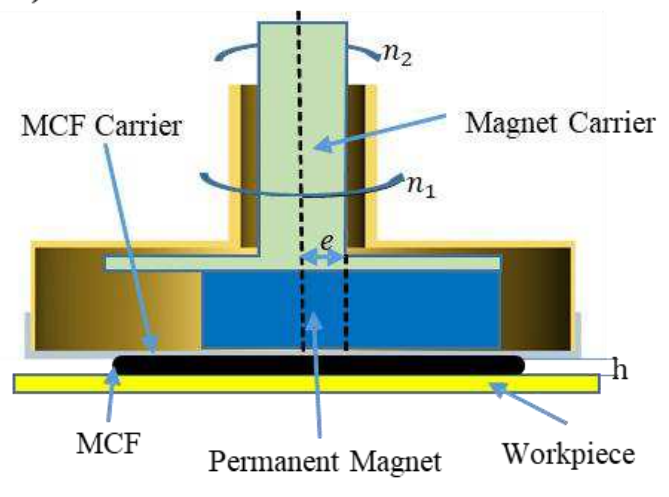

Fig. 2 a) MCF finishing experimental platform. b) Diagram of finishing head 


\subsection{Determination of KPPs and experimental design}

Taking all parameters into consideration while developing a machining model is time-consuming and unnecessary. Therefore, preliminary experiments were conducted based on previous research results to determine the key finishing parameters (KFPs), which have the greatest influence on the finishing effect and need to be optimized. Finally, the abrasive particle size (s), machining gap (h), MCF carrier rotational speed (n), and finishing time (t) were selected as KFPs in modeling. The remaining parameters were fixed to their appropriate values. For example, the function of the rotational magnetic is to provide a dynamic magnetic field whose state has a relatively small effect on the finishing effect. Therefore, the magnet rotates in the opposite direction of the MCF carrier, and the speed is maintained at $100 \mathrm{rpm}$. Each KFP is divided into five levels. The machining gap, MCF carrier rotational speed, and finishing time are uniformly distributed, but the abrasive particle size cannot be uniformly distributed since it can only be selected according to the existing sizes. The sizes of abrasive particles are 4000 \#, 5000 \#, 8000 \#, 10000 , and 12500 \#. The corresponding abrasive particle diameters are $3.4 \mu \mathrm{m}, 2.6 \mu \mathrm{m}, 1.6 \mu \mathrm{m}, 1.3 \mu \mathrm{m}, 1 \mu \mathrm{m}$, respectively. The levels setting of each KFP is shown in Table 2 .

Table 2 Levels of KFPs

\begin{tabular}{cccccc}
\hline KFPs & \multicolumn{5}{c}{ Levels } \\
\hline Abrasive particle size $(\mu \mathrm{m})$ & 1 & 1.3 & 1.6 & 2.6 & 3.4 \\
Machining gap $(\mathrm{mm})$ & 1 & 1.25 & 1.5 & 1.75 & 2 \\
MCF carrier rotational speed (rpm) & 200 & 400 & 600 & 800 & 1000 \\
Finishing time $(\mathrm{min})$ & 30 & 60 & 90 & 120 & 150 \\
\hline
\end{tabular}

\subsection{Design and development of experiments}

Copper alloys (H62) with a fixed size $(50 \mathrm{~mm} \times 50 \mathrm{~mm} \times 1 \mathrm{~mm})$ were used as the experimental workpieces. The surface roughness of the workpiece was pre-polished to $0.2 \mu \mathrm{m}$ for minimizing the effect of the workpiece's initial surface roughness on the polishing effect. Due to the difficulty of quantifying the workpiece's dimensional accuracy, the amount of material removed was utilized to indicate the reduction in dimensional accuracy. The more material removed, the more effect finishing has on dimensional precision.

The experiment was designed without considering the time levels using the Design-Expert software. Ra and MRA were measured every 30 minutes in the actual experiment. Ra was measured with the TR200 surface roughness instrument, and the MRA was measured with high precision electronic analysis balance. Eight spots on each sample surface were assessed, and the average value was used to determine the final Ra of the sample surface. Some experimental data are shown in Table 3.

Table 3 Plan of experiments and response summary

\begin{tabular}{|c|c|c|c|c|c|c|c|c|c|}
\hline \multirow{2}{*}{ NO. } & \multirow{2}{*}{$\mathrm{s}(\mu \mathrm{m})$} & \multirow{2}{*}{$\mathrm{h}(\mathrm{mm})$} & \multirow{2}{*}{$\mathrm{n}(\mathrm{rpm})$} & & \multicolumn{5}{|c|}{$\mathrm{t}(\mathrm{min})$} \\
\hline & & & & & 30 & 60 & 90 & 120 & 150 \\
\hline \multirow{2}{*}{1} & \multirow{2}{*}{3.4} & \multirow{2}{*}{2} & \multirow{2}{*}{200} & $\mathrm{Ra}(\mu \mathrm{m})$ & 0.082 & 0.073 & 0.063 & 0.054 & 0.045 \\
\hline & & & & $\operatorname{MRA}(\mathrm{g})$ & 0.0353 & 0.0522 & 0.0853 & 0.1875 & 0.2701 \\
\hline \multirow{2}{*}{2} & \multirow{2}{*}{2.6} & \multirow{2}{*}{1.5} & \multirow{2}{*}{600} & $\mathrm{Ra}(\mu \mathrm{m})$ & 0.065 & 0.048 & 0.041 & 0.039 & 0.038 \\
\hline & & & & MRA (g) & 0.0558 & 0.1162 & 0.1806 & 0.2289 & 0.2680 \\
\hline$\cdots$ & $\cdots$ & $\cdots$ & $\cdots$ & $\cdots$ & $\ldots$ & $\cdots$ & $\cdots$ & $\cdots$ & $\cdots$ \\
\hline \multirow{2}{*}{29} & \multirow{2}{*}{1} & \multirow{2}{*}{2} & \multirow{2}{*}{600} & $\mathrm{Ra}(\mu \mathrm{m})$ & 0.092 & 0.080 & 0.071 & 0.061 & 0.054 \\
\hline & & & & $\operatorname{MRA}(\mathrm{g})$ & 0.0376 & 0.0536 & 0.0670 & 0.0849 & 0.1546 \\
\hline \multirow{2}{*}{30} & \multirow{2}{*}{2.6} & \multirow{2}{*}{1} & \multirow{2}{*}{600} & $\mathrm{Ra}(\mu \mathrm{m})$ & 0.056 & 0.042 & 0.035 & 0.032 & 0.033 \\
\hline & & & & $\operatorname{MRA}(\mathrm{g})$ & 0.0893 & 0.1709 & 0.2473 & 0.2824 & 0.2988 \\
\hline
\end{tabular}




\section{Modeling and Validation}

\subsection{Establishment of machining model}

The accuracy of the machining model has a significant impact on the optimization of finishing parameters. In machining and manufacturing, principle modeling and mathematical fitting are two standard modeling methods. However, it is difficult to obtain a sufficiently accurate prediction model through principle modeling because of the complicated formation mechanism MCFF and the complex interaction between MCF and the workpiece surface. Additionally, It is challenging to achieve high prediction accuracy using single mathematical fitting methods, such as response surface and polynomial fitting. Therefore, an MNNFM with higher prediction accuracy was built in this research. By fuse the predictions from several models, the fusion model can minimize prediction variance and provide more accurate predictions than a single model. The process is known as ensemble learning.

As shown in Fig. 3, the established MNNFM is composed of two layers: a prediction layer and a fusion layer.

In the prediction layer, five different neural network models with excellent prediction performance, which were selected from different types of neural network models, are used to predict Ra and MRA. Each neural network model is an independent predictor with four inputs and two outputs. Four inputs represent the four finishing parameters that need to be optimized, and two outputs represent Ra and MRA. The structural parameters of each neural network are shown in Table 4.

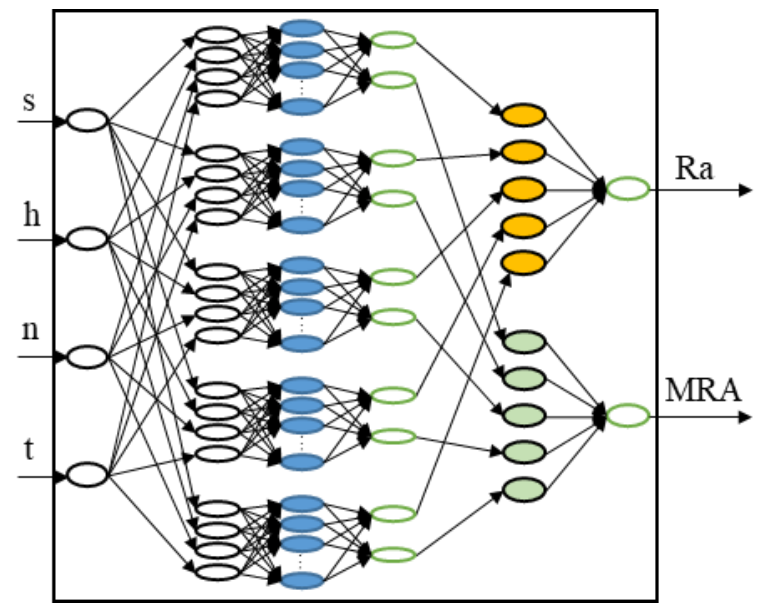

Fig. 3 The structure of the MNNFM

Table 4 The parameters of prediction layer neural network

\begin{tabular}{cccccc}
\hline Net & Number of hidden layers & Number of nodes & Training algorithm & Activation function & $\mathrm{R}^{2}$ \\
\hline 1 & 1 & 10 & Elastic BP algorithm & Tanh & 0.923 \\
2 & 1 & 8 & Polak-Ribiers conjugategradient algorithm & Sigmoid & 0.935 \\
3 & 1 & 9 & Powell-Beale conjugategradient algorithm & Identity & 0.925 \\
4 & 1 & 8 & BFGS Quasi newton method & Identity & 0.907 \\
5 & 1 & 10 & Levenberg - Marquardt algorithm & Sigmoid & 0.949 \\
\hline
\end{tabular}

The function of the fusion layer is to obtain more accurate prediction results based on the output of each neural network in the prediction layer. The fusion layer may alternatively be a neural network that accepts Ra and MRA from the prediction layer's output as input and outputs Ra and MRA. However, in order to simplify the computation, a weighting strategy was adopted to fuse the output of the prediction layer in this study. As shown in Eq. (1), the Ra output by MNNFM is the weighted sum of the Ra output by the five neural network models in the prediction layer, and the same is true for MRA. The weight of each neural network model is 
determined by its regression coefficient. The weight and influence of the neural network model on MNNFM output increase with the regression coefficient increase.

$$
\begin{aligned}
& R a=\sum_{i=1}^{5} R a_{i} W_{i} \\
& M R A=\sum_{i=1}^{5} M R A_{i} W_{i} \\
& W_{i}=\frac{R_{i}^{2}}{\sum_{i=1}^{5} R_{i}^{2}}
\end{aligned}
$$

\subsection{Verification of model prediction performance}

Although the regression coefficients can reflect the predictive performance of the established model to a certain extent, it is worthwhile to further compare and verify the predictive performance of the established model through specific examples. Six sets of finishing parameters are randomly created within the feasible area, and the developed MNNFM was utilized to forecast Ra and MRA. Simultaneously, the prediction results of the prediction layer's five neural networks were recorded for comparison. Finally, the verification experiments were conducted to validate the model's prediction performance. The results are illustrated in Fig. 4.

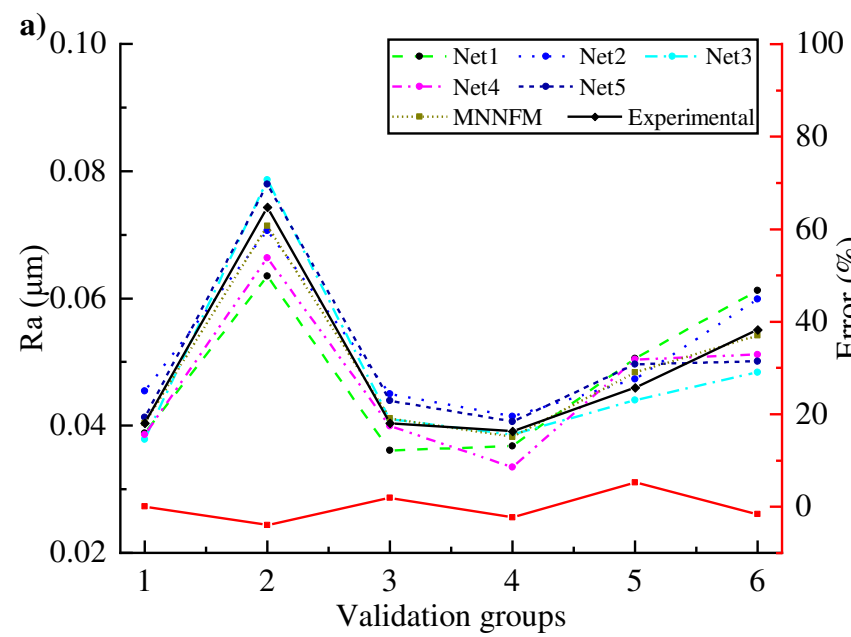

Fig. 4 Verification of the MNNFM

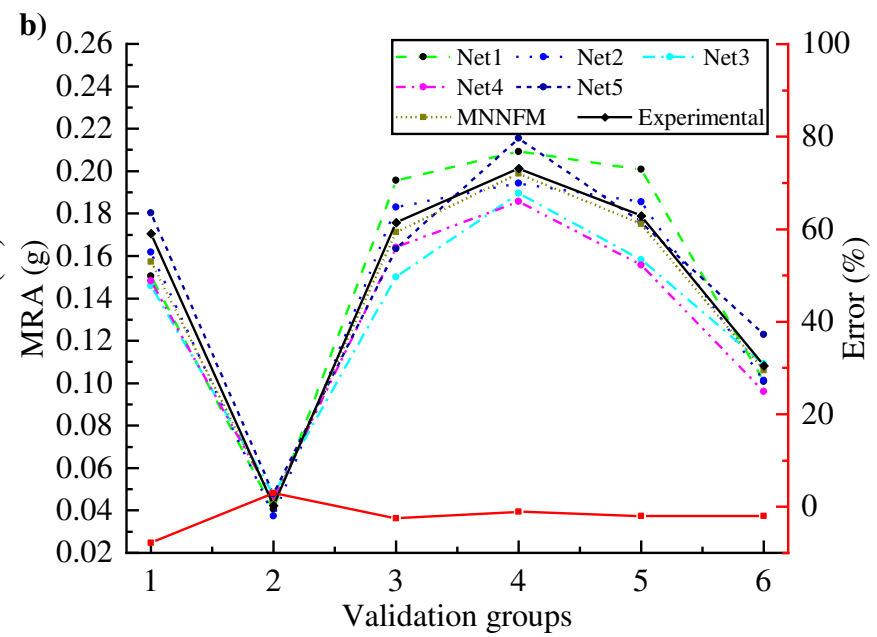

b) MRA

It can be found from Fig. 4 that compared with the prediction results of a single model, the prediction results of MNNFM for $\mathrm{Ra}$ and MRA are generally closer to the experimental results. This means that the prediction accuracy of MNNFM for Ra and MRA is higher than that of any single model. While a single model may outperform MNNFM at certain points, the model should be evaluated holistically rather than at particular points. Additionally, a single model may have a high forecast deviation at certain points, while MNNFM provides excellent prediction performance at all points and reduces the possibility of large prediction deviations. MNNFM has the capacity to compensate for a single model's prediction mistakes, which may explain why MNNFM has superior prediction performance. For instance, the MRAs predicted by the second neural network model for the second and fourth sets of finishing parameters are considerably higher than the experimental values. On the contrary, the third neural network model's prediction results are considerably lower than the experimental values. Larger prediction errors may occur when any single model is adopted, resulting in an inability to perform an accurate assessment of the finishing parameters during parameter optimization. When the two models are combined for MRA prediction, better prediction accuracy is possible. As shown in Fig. 4b), the MNNFM prediction results for the second and fourth sets of finishing parameters are more consistent with the experimental values. Increased 
prediction accuracy will aid in accurately evaluating the finishing parameters throughout the optimization process, resulting in the most optimum finishing parameters.

The correlation coefficient of the established MNNFM is 0.989 , which is considerably higher than the correlation coefficient of other single models. Additionally, the MNNFM prediction error percentages for Ra and MRA are within 10\%, which is considered acceptable in this research. In general, the developed MNNFM performs well in terms of forecast accuracy, and its prediction outputs may accurately represent actual processing conditions. Therefore, the subsequent parameter optimization and the analysis of the finishing process are based on the established MNNFM.

\section{Optimization of finishing parameters}

\subsection{Process of parameter optimization}

It is unavoidable that material removal during the finishing process may affect the workpiece's dimension accuracy. However, excessive material removal significantly lowers the dimensional accuracy, which is not acceptable. When just the Ra of the polished surface is considered without regard for the MRA, the dimensional accuracy may be compromised. For instance, after 150 minutes of finishing with the thirties set of finishing parameters in Table 3, the MRA is about $0.3 \mathrm{~g}$. Based on the surface dimensions of the workpiece (L $50 \mathrm{~mm}$, W $50 \mathrm{~mm}$ ) and the density of copper H62 $\left(8.43 \mathrm{~g} / \mathrm{cm}^{3}\right)$, it is estimated that the thickness of the sample will decrease by about $14 \mu \mathrm{m}$, which is unacceptable in high-precision workpieces. Therefore, it is necessary to take both Ra and MRA into account when optimizing finishing parameters.

In the case of optimizing finishing parameters while taking the effect of MRA on dimensional accuracy into account, the mathematical model is represented by Eq. (2). There are two optimization objectives: minimizing Ra and minimizing MRA. Additionally, each parameter's value must be maintained within an acceptable range.

$$
\begin{aligned}
& f_{1}: \min R a=F_{1}(s, h, n, t) \\
& f_{2}: \min M R A=F_{2}(s, h, n, t) \\
& \text { s.t. } \\
& s \in\left[\begin{array}{lllll}
1 & 1.3 & 1.6 & 2.6 & 3.4
\end{array}\right] \\
& 1 \leq h \leq 2 \\
& 200 \leq n \leq 1000 \\
& 30 \leq t \leq 150
\end{aligned}
$$

The MOPSO algorithm was employed to optimize the finishing parameters because of its outstanding performance in multiobjective optimization. Fig. 5 shows the pseudocode of the MOPSO algorithm. The MOPSO starts with N randomly generated particles (finishing parameters), where each particle has position and velocity properties. In this study, the position and velocity of each particle are four dimensions, respectively representing the four finishing parameters to be optimized. $x_{i}^{t}=\left\{W_{i}^{t}, h_{i}^{t}, n_{i}^{t}, t_{i}^{t}\right\}$ and $v_{i}^{t}=\left\{v_{w_{i}}^{t}, v_{h_{i}}^{t}, v_{n_{i}}^{t}, v_{t_{i}}^{t},\right\}$ represent position and velocity of particle $i=1, \ldots, N$ at iteration $t=1, \ldots, t_{\text {max }}$, respectively. The initial position and velocity are random values within the acceptable range. Then, each particle is evaluated based on the established MNNFM. Particle $i$ will be determined to be a non-dominated optimal solution and stored in an external repository (REP) if there is no other particle in the entire population that has a better surface and less MRA than particle $i$. At each iteration of the MOPSO, the obtained non-dominant solutions are compared one by one to the solutions in REP. If the new solution is dominated by any member of REP, the new solution will be discarded; otherwise, the new solution will be added to REP. If there are any solutions in the REP dominated by the new solution, the former will be discarded. When the number of solutions in REP exceeds the limit, an adaptive grid is required to remove excess solutions. The target space is divided into several small regions. If there are multiple solutions in a region, some redundant solutions in the region will be randomly discarded. This allows the final 
goal to have well-distributed non-dominant solutions [27].

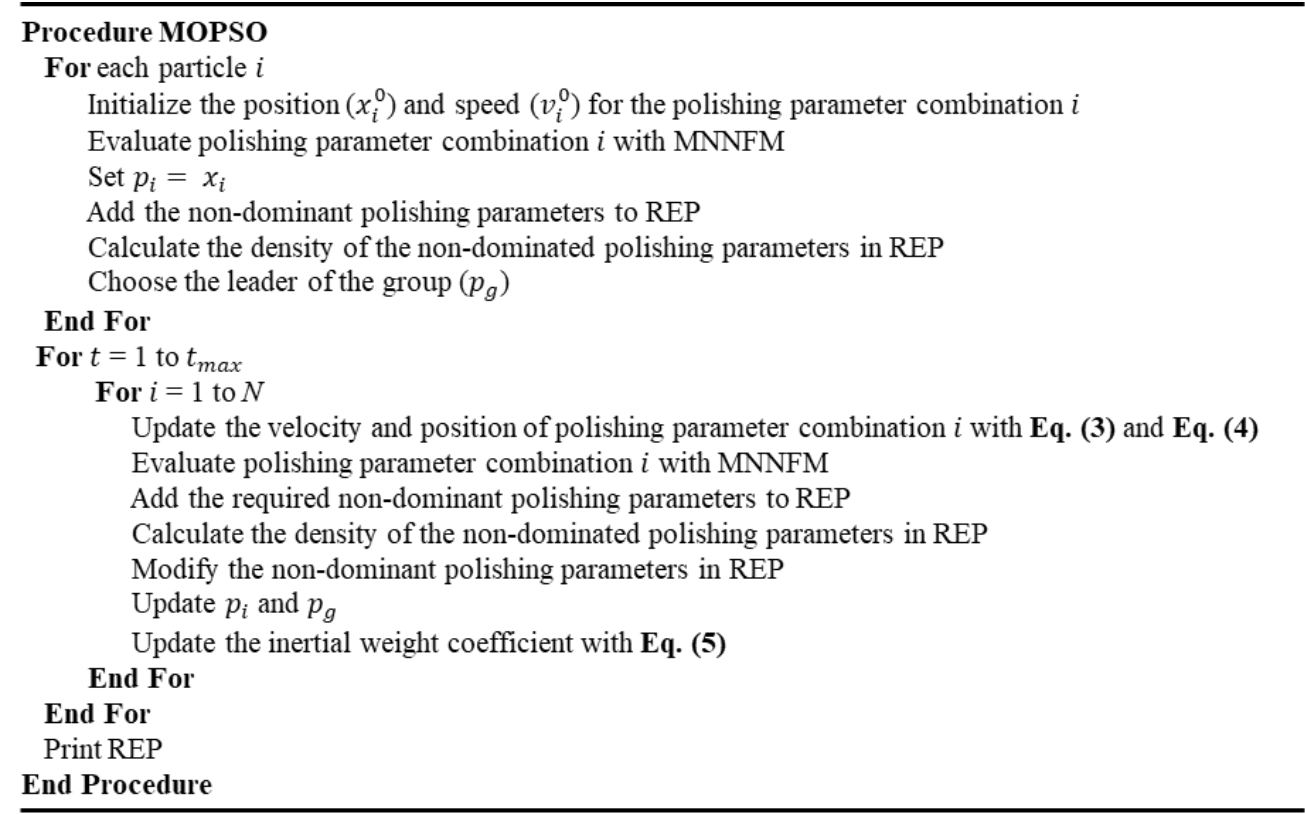

Fig. 5 Pseudocode of MOPSO

In the optimization process of MOPSO, each particle continually updates its velocity and position by Eq. (3) and Eq. (4). $p_{i}$ represents the best position of each particle, $p_{g}$ represents the position of the best population leader. Additionally, the location of the best particle can not be found directly. Instead, the crowding of the solutions in the REP will be calculated, and the solutions with lower crowding are more likely to be selected as the best population leader.

$$
\begin{gathered}
v_{i}^{t+1}=w^{t} v_{i}^{t+1}+c_{1} r_{1}\left(p_{i}-x_{i}^{t}\right)+c_{2} r_{2}\left(p_{g}-x_{i}^{t}\right) \\
x_{i}^{t+1}=x_{i}^{t}+v_{i}^{t+1}
\end{gathered}
$$

Where $r_{1}$ and $r_{2}$ are uniformly distributed random numbers between 0 and 1 , and $c_{1}$ and $c_{2}$ are learning factors; $w^{t}$ represents the inertial weight coefficient and controls the tradeoff between the global and local history, which is calculated by Eq. (5):

$$
w^{t}=w_{\max }-\frac{t\left(w_{\max }-w_{\min }\right)}{t_{\max }}
$$

The number of non-dominated optimal solutions that can be stored in REP can be adjusted according to actual needs. The REP can store up to 50 sets of non-dominated optimal solutions in this research. This means that 50 sets of excellent finishing parameters will be obtained after finishing, which are the results of balancing Ra and MRA. Table 5 shows part of the non-dominant solutions recorded in REP. The complete non-dominant solutions are shown in Appendix A. The finishing parameters listed in Appendix A can provide a high-quality surface while minimizing material removal and ensuring dimensional accuracy.

Table. 5 Non-dominated optimal solutions recorded in REP

\begin{tabular}{ccccccc}
\hline NO. & $\mathrm{s}(\mu \mathrm{m})$ & $\mathrm{h}(\mathrm{mm})$ & $\mathrm{n}(\mathrm{rpm})$ & $\mathrm{t}(\mathrm{min})$ & $\mathrm{Ra}(\mu \mathrm{m})$ & $\mathrm{MRA}(\mathrm{g})$ \\
\hline 1 & 3.4 & 1 & 1000 & 150 & 0.0304 & 0.2753 \\
& & & 9 & & &
\end{tabular}




\begin{tabular}{ccccccc}
2 & 3.4 & 1 & 860 & 131 & 0.0314 & 0.2546 \\
$\ldots$ & $\ldots$ & $\ldots$ & $\ldots$ & $\ldots$ & $\ldots$ & $\ldots$ \\
49 & 1.6 & 2 & 200 & 36 & 0.0714 & 0.0312 \\
50 & 1.3 & 2 & 200 & 30 & 0.0807 & 0.0272 \\
\hline
\end{tabular}

\subsection{Discussion of optimization results}

By analyzing the non-dominated optimal solutions recorded in REP, it can be found that: 1) When the high-quality polished surface is required, the finishing parameters adopted have a larger abrasive particle size, smaller machining gap, faster MCF carrier rotational speed, and longer finishing time; 2) When the quality requirement for the polished surface is not strict, the finishing parameters used have a medium size, larger machining gap, lower MCF carrier rotational speed, and shorter finishing time. In order to explore the reasons, the influences of the finishing parameters on Ra and MRA were studied, and the obtained results are shown in Fig. 6.

a)

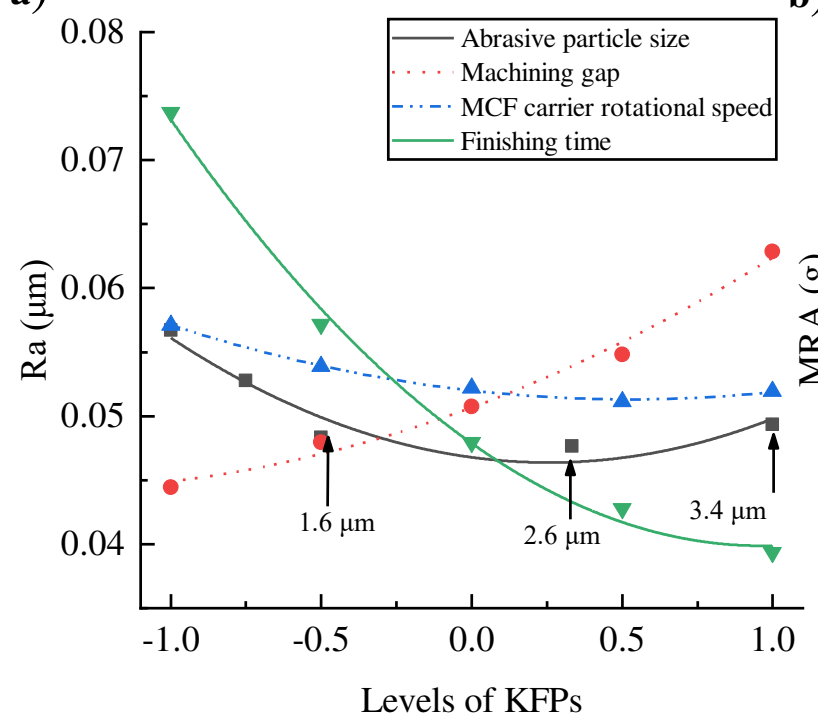

b)

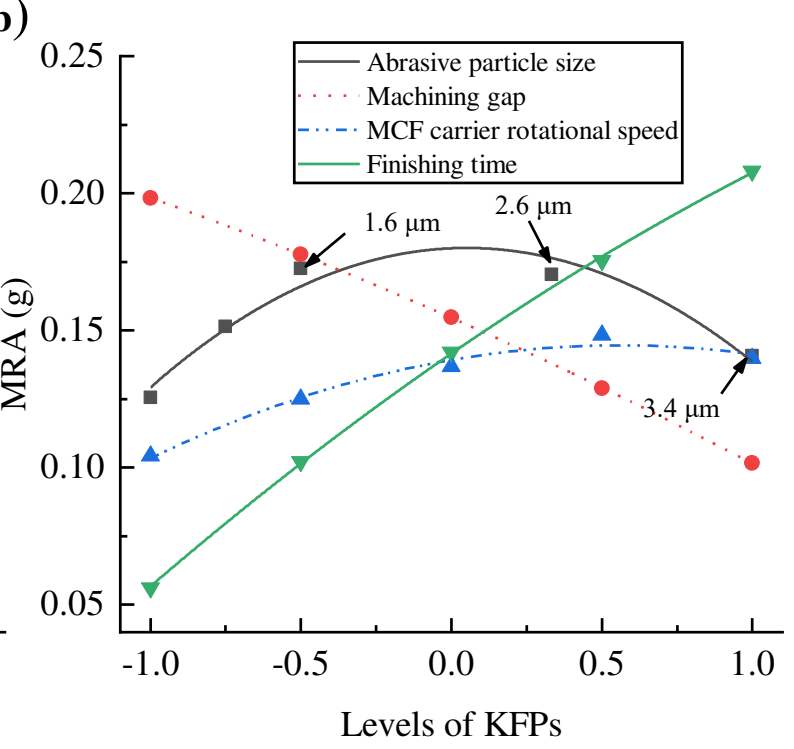

Fig. 6 a) Effect of KFPs on Ra b) Effect of KFPs on MRA

As shown in Fig. 6, the finishing time is the most influential parameter on Ra and MRA, followed by machining gap and abrasive particle size. With the increase of time, Ra shows a decreasing trend. Further, it can be found that the rate of decline decreased as time passed. The reason behind this is that the initial material surface is rough and contains many readily removed micro bulges, resulting in a rapid decrease in Ra. In the later stage, the material surface becomes smooth. While removing the microprotrudes, the abrasive particles will also break the smooth surface, resulting in a slower decreasing rate of Ra. Therefore, when the required surface quality of the workpiece is high, long-time finishing is difficult to avoid. However, it is not prudent to raise the finishing time blindly. After the workpiece's surface quality reaches a certain point, increasing the finishing time slightly improves the workpiece's surface quality while significantly increasing the time cost and material removal.

The working gap has a significant impact on the finishing effect since it alters the finishing force. The smaller the machining gap, the greater the finishing force, and vice versa. With a smaller working gap, the magnet is closer to the workpiece's surface, the magnetic cluster produced is more stable, exerting more force on the workpiece's surface. Additionally, the amount of MCF flowing through the machining gap per unit time is constant at a particular MCF carrier rotational speed. A reduced machining gap results in an increase in hydrodynamic pressure on the workpiece's surface, resulting in increased finishing force. As shown in Fig. 6a), the workpiece's surface quality declines as the machining gap grows. This is because the tiny shear is insufficient to remove the material, 
and more elastic deformation occurs on the material's surface, making it difficult to improve the workpiece's surface quality. The increased shearing force will remove material effectively and improve the workpiece's surface quality. Therefore, a smaller machining gap will be selected to get a high-quality polished surface. However, smaller machining gaps also mean greater material removal. A larger machining gap was selected when the quality of the polished surface was not demanding.

Abrasive particles are the main contributor to material removal. The abrasive particles are held by multiple CIPs in the FMAB, and the clamping force of the two CIPs on the abrasive particles changes with the size of the abrasive particles. The study by Huang et al. showed that the large size of abrasive particles generate higher clamping force when the size of CIPs is the same as that of abrasive particles[28]. Simultaneously, larger abrasive particle size increases the contact area between the abrasive particles and the workpiece, resulting in an increase in the force required for the abrasive particles to be pressed into the workpiece's surface. Due to the presence of two distinct effects, MRA increases first and subsequently decreases with the rise of abrasive particle size. Correspondingly, Ra shows a trend of decreasing first and then increasing. Therefore, when high-quality surfaces are required, the optimal abrasive particle size should be $2.6 \mu \mathrm{m}$ if the dimensional accuracy is ignored. However, the optimized abrasive particle size is $3.4 \mu \mathrm{m}$ when the dimensional accuracy is taken into consideration. This is mainly because the increase in Ra is not obvious, while the decrease in MRA is obvious when the abrasive particle size increases from $2.6 \mu \mathrm{m}$ to $3.4 \mu \mathrm{m}$. When surface quality requirements are not strict, abrasive particles are typically $1.6 \mu \mathrm{m}$ in size, which can strike a balance between surface quality and dimensional accuracy.

The MCF carrier rotational speed has a small effect on Ra and MRA. When the MCF carrier rotational speed is increased, the speed of the abrasive particles rises as well, as does the force produced by the abrasive particles on the workpiece's surface. In general, Ra decreases as the speed rises, whereas MRA increases. However, when the speed is excessive, Ra starts to exhibit an insignificant upward trend, and MRA exhibit an insignificant downward trend. This phenomenon may be caused by the increase of the centrifugal force of the abrasive particles due to the excessive speed, which increases the probability of the abrasive particles escaping from the FMAB and reduces the finishing efficiency. As a result, a large MCF carrier rotational speed is required when the surface quality requirements are high. When the surface quality requirements are not strict, a low speed may satisfy the requirements and reduce MRA.

The above part discusses the distribution of the non-dominant optimal solutions derived via an investigation of the effect of finishing parameters on Ra and MRA. The following part will illustrate the superiority of the non-dominated optimal solutions discovered through comparison.

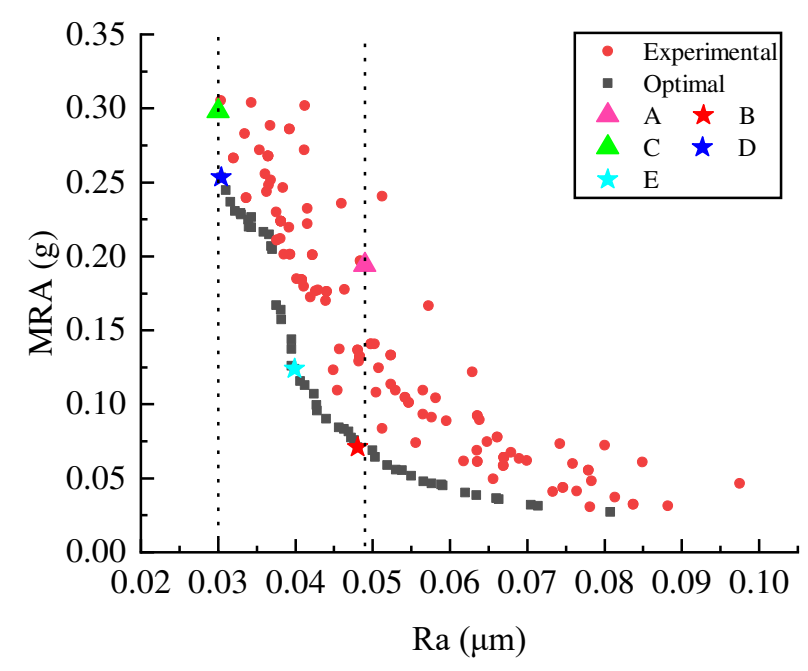

Fig. 7 Optimization results considering dimensional accuracy

Ra and MRA corresponding to the non-dominated optimal solutions are offered in Fig. 7. In addition, the results of the previous experiment are also shown in Fig. 7. It can be found that the optimized Ra and MRA tend to the lower-left corner, which means that 
the workpiece with high-quality, low MRA and high dimensional accuracy can be obtained using the optimized finishing parameters. For instance, to obtain polished surfaces with surface roughness less than $0.05 \mu \mathrm{m}$, the Ra obtained using the optimal finishing parameters in the previous experiment is $0.049 \mu \mathrm{m}$, and the MRA is $0.1970 \mathrm{~g}$ (Point A in Fig. 7). When the finishing parameters are selected from the non-dominant solutions, the obtained Ra is $0.049 \mu \mathrm{m}$, but the MRA is only $0.0712 \mathrm{~g}$ (Point B in Fig.7). The MRA is reduced by about $63.9 \%$. In addition, the finishing time is also reduced from $90 \mathrm{~min}$ to $55 \mathrm{~min}$, and finishing efficiency is improved. Assuming that the material is uniformly removed, the thickness reduction of the workpiece decreases from $9.348 \mu \mathrm{m}$ to $3.378 \mu \mathrm{m}$. This means that the dimensional accuracy is improved by $63.9 \%$ with the optimized finishing parameters.

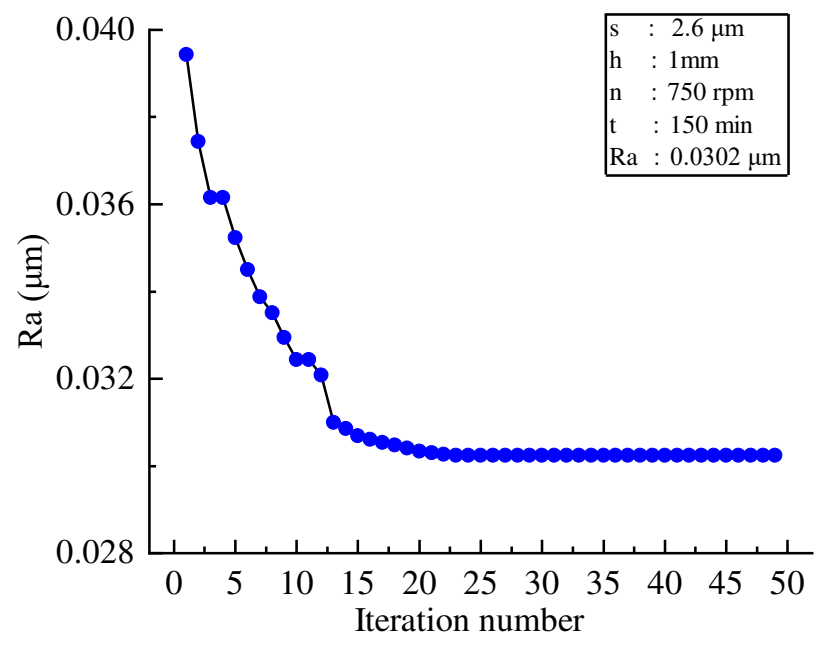

Fig.8 Optimization iterative process without considering dimensional accuracy

To compare the optimization results of finishing parameters with considering dimensional accuracy and without considering dimensional accuracy, the particle swarm optimization algorithm was employed to optimize the finishing parameters without considering dimensional accuracy. The obtained iteration process and finishing parameters are shown in Fig. 8. The Ra obtained by using the finishing parameters in Fig. 8 is $0.0302 \mu \mathrm{m}$, and the MRA is $0.2982 \mathrm{~g}$ (Point C in Fig. 7). However, the smallest Ra that can be obtained using the finishing parameters optimized considering the dimensional accuracy is $0.0304 \mu \mathrm{m}$, which is almost the same as the Ra obtained without considering the dimensional accuracy, and the obtained MRA is reduced to $0.2533 \mathrm{~g}$ (Point $\mathrm{D}$ in Fig. 7). The amount of material removed was decreased by $15.1 \%$. This demonstrates that even when a highly polished surface is requested, the proposed optimization algorithm for finishing parameters may successfully minimize MRA, and maintaining the workpiece's dimension accuracy.

Additionally, the improvement of the workpiece's dimensional accuracy by the proposed optimization algorithm of finishing parameters will be more obvious when the demands for the workpiece's surface quality are not strict. Under the condition that the workpiece with Ra less than $0.0400 \mu \mathrm{m}$ can meet the requirements of use, the polished surface with Ra of $0.0399 \mu \mathrm{m}$ can be obtained by using the finishing parameters in the non-dominant optimal solution, and the corresponding MRA is $0.1240 \mathrm{~g}$ (Point E in Fig. 7). If the optimized finishing parameters without considering dimensional accuracy are adopted, the quality of the polished surface exceeds the need, but this results in an increase in MRA and a reduction in the workpiece's dimensional accuracy. MRA will be decreased by $58.4 \%$ using the optimized finishing parameters considering dimensional accuracy, which means that the workpiece's dimensional accuracy will be improved by $58.4 \%$.

The above study demonstrates that the finishing parameters obtained by the proposed finishing parameter optimization method may minimize material removal and enhance the workpiece's dimensional accuracy while obtaining a high-quality polished surface. 


\subsection{Validation of optimization results}

To validate the optimization results, validation experiments were done on both optimization results without considering MRA and the optimization results with considering MRA. Each group of experiments was repeated three times. Fig. 9 shows the comparison between the experimental results and the predicted results. It can be found that the predicted results are close to the experimental results.

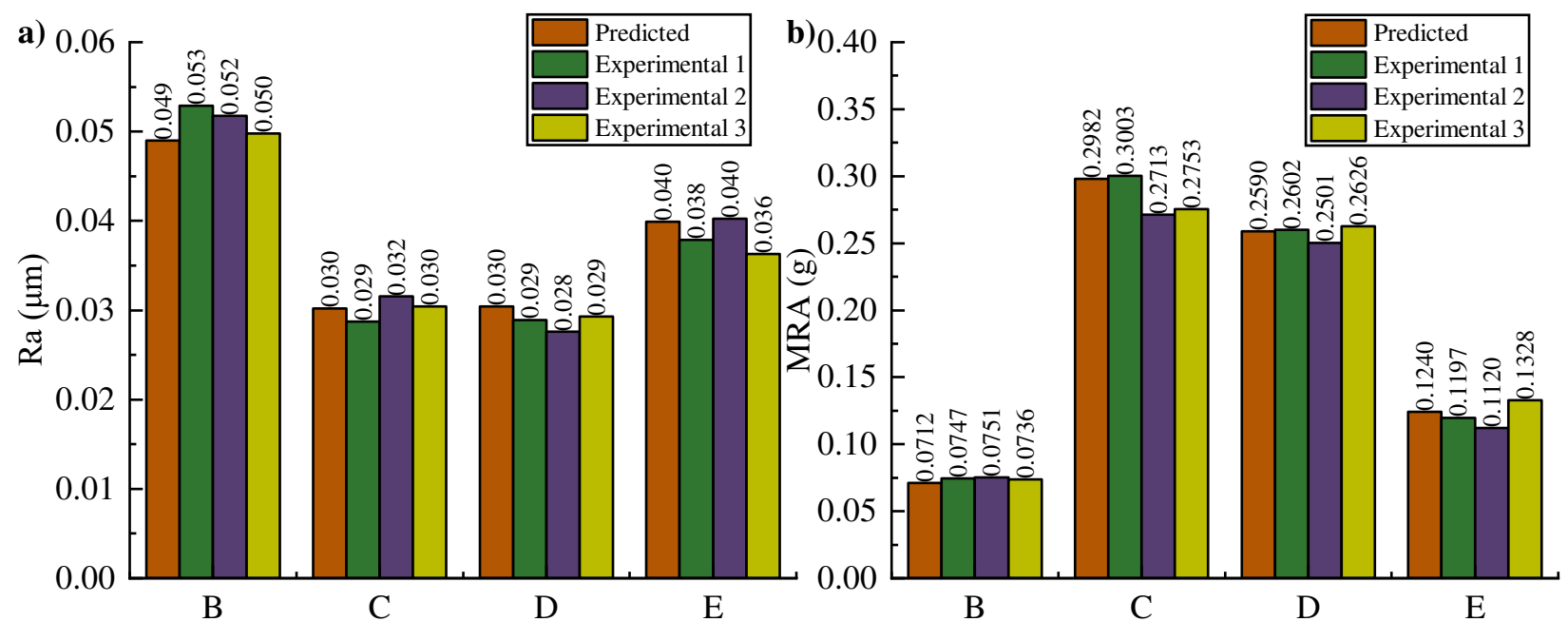

Fig. 9 Verification of optimization results

Table 6 Verification of optimization results

\begin{tabular}{|c|c|c|c|c|c|c|c|c|c|c|}
\hline \multirow{2}{*}{ Points } & \multirow{2}{*}{$\mathrm{s}(\mu \mathrm{m})$} & \multirow{2}{*}{$\mathrm{h}(\mathrm{mm})$} & \multirow{2}{*}{$\mathrm{n}(\mathrm{rpm})$} & \multirow{2}{*}{$\mathrm{t}(\min )$} & \multicolumn{2}{|c|}{ Predictions } & \multicolumn{2}{|c|}{ Experiments } & \multirow{2}{*}{\multicolumn{2}{|c|}{ Error $(\%)$}} \\
\hline & & & & & $\mathrm{Ra}(\mu \mathrm{m})$ & MRA (g) & $\mathrm{Ra}(\mu \mathrm{m})$ & MRA (g) & & \\
\hline B & 1.6 & 1.8 & 210 & 55 & 0.049 & 0.0712 & 0.0517 & 0.0745 & $-5.22 \%$ & $-4.43 \%$ \\
\hline $\mathrm{C}$ & 2.6 & 1 & 750 & 150 & 0.0302 & 0.2982 & 0.0303 & 0.2858 & $-0.33 \%$ & $4.34 \%$ \\
\hline $\mathrm{D}$ & 3.4 & 1 & 1000 & 150 & 0.0304 & 0.2533 & 0.0287 & 0.2580 & $5.92 \%$ & $-1.84 \%$ \\
\hline $\mathrm{E}$ & 1.6 & 1.7 & 370 & 84 & 0.0399 & 0.1240 & 0.0381 & 0.1215 & $4.72 \%$ & $2.05 \%$ \\
\hline
\end{tabular}

Table 6 shows the mean value of the three experimental values and the deviation between the predicted and experimental results. It can be found that the prediction errors of the optimized finishing parameters are less than $10 \%$. This verifies the accuracy of the established MNNFM and the optimization results again. The proposed modeling method and process parameter optimization method are demonstrated to play an excellent role in the MCF finishing process. The proposed multi-objective optimization method can significantly decrease the roughness of the polished surface while maintaining dimensional accuracy.

\section{Conclusion}

The aim of the present research was to determine the optimal finishing parameters for copper alloys in order to obtain the workpieces with a high-quality surface and high dimensional accuracy. The finishing model was established and the finishing parameters were optimized. The main conclusions are summarized as follows:

- The finishing time has the greatest effect on Ra and MRA, followed by the machining gap and abrasive particle size, while the MCF carrier rotational speed has the least effect on Ra and MRA.

- Although a high-quality polished surface usually requires more material removal during the finishing process, the optimum balance between them may be achieved by utilizing the appropriate finishing parameters. 
- In the pursuit of the highest quality polished surface, a slight sacrifice of finishing quality may bring a considerable increase in dimensional accuracy by using optimized finishing parameters.

- In the case of low finishing quality requirements, taking the effect of material removal on dimensional accuracy into consideration when optimizing finishing parameters may significantly improve the workpiece's dimensional accuracy.

\section{Funding}

This work was supported by the National Key Research and Development Project (Grant numbers 2018YFB1107502) and the National Natural Science Foundation of China (Grant numbers 51705011).

\section{Author's contribution}

Jinwei Fan and Xingfei Ren provided ideas for this study, wrote codes and manuscripts. Ri Pan assisted in the revision of the article and the compilation of codes. Peitong Wang and Haohao Tao were responsible for the experiment in this study. All authors contributed to this study.

\section{Availability of data and material}

The authors confirm that the data supporting the findings of this study are available within the article.

\section{Code availability}

The authors confirm that the code supporting the findings of this study are available within the article.

\section{Ethics declarations}

\section{Ethics approval}

The research does not involve ethical issues.

\section{Consent for publication}

All the authors agreed to publish this paper.

\section{Competing interests}

The authors declare no competing interests.

\section{References}

1. Ein-Eli, Y., \& Starosvetsky, D. (2007) Review on copper chemical-mechanical polishing (CMP) and post-CMP cleaning in ultra large system integrated (ULSI)—An electrochemical perspective. Electrochimica Acta 52(5):1825-1838. https://doi.org/10.1016/j.electacta.2006.07.039

2. Khan, D. A., \& Jha, S. (2019) Selection of optimum polishing fluid composition for ball end magnetorheological finishing (BEMRF) of copper. The International Journal of Advanced Manufacturing Technology 100(5):1093-1103. https://doi.org/10.1007/s00170-017-1056-9

3. Pan, B., Kang, R., Guo, J., Fu, H., Du, D., \& Kong, J. (2019) Precision Fabrication of Thin Copper Substrate by Doublesided Lapping and Chemical Mechanical Polishing. Journal of Manufacturing Processes 44:47-54. https://doi.org/10.1016/j.jmapro.2019.05.035

4. Das, M., Jain, V. K., \& Ghoshdastidar, P. S. (2012) Nanofinishing of flat workpieces using rotational-magnetorheological abrasive flow finishing (R-MRAFF) process. International Journal of Advanced Manufacturing Technology 62(1-4):405420. https://doi.org/10.1007/s00170-011-3808-2

5. Jha, S., \& Jain, V. K. (2004) Design and development of the magnetorheological abrasive flow finishing (MRAFF) process. International Journal of Machine Tools and Manufacture 44(10):1019-1029. https://doi.org/10.1016/j.ijmachtools.2004.03.007

6. Jiao, L., Wu, Y., Wang, X., Guo, H., \& Liang, Z. (2013) Fundamental performance of Magnetic Compound Fluid (MCF) wheel in ultra-fine surface finishing of optical glass. International Journal of Machine Tools and Manufacture 75:109-118. 
https://doi.org/10.1016/j.ijmachtools.2013.09.003

7. Alam, Z., \& Jha, S. (2017) Modeling of surface roughness in ball end magnetorheological finishing (BEMRF) process. Wear 374-375:54-62. https://doi.org/10.1016/j.wear.2016.11.039

8. DeGroote, J. E., Marino, A. E., Wilson, J. P., Bishop, A. L., Lambropoulos, J. C., \& Jacobs, S. D. (2007) Removal rate model for magnetorheological finishing of glass. Applied Optics 46(32):7927-7941. https://doi.org/10.1364/A0.46.007927

9. Vahdati, M., \& Rasouli, S. A. (2016) Study of magnetic abrasive finishing on freeform surface. Transactions of the IMF 94(6):294-302. https://doi.org/10.1080/00202967.2016.1232822

10. Sidpara, A., \& Jain, V. K. (2012) Nano-level finishing of single crystal silicon blank using magnetorheological finishing process. Tribology International 47:159-166. https://doi.org/10.1016/j.triboint.2011.10.008

11. Niranjan, M., Jha, S., \& Kotnala, R. K. (2014) Ball End Magnetorheological Finishing Using Bidisperse Magnetorheological Polishing Fluid. Materials and Manufacturing Processes 29(4):487-492. https://doi.org/10.1080/10426914.2014.892609

12. Guo, H., Wu, Y., Lu, D., Fujimoto, M., \& Nomura, M. (2014b) Ultrafine Polishing of Electroless Nickel-Phosphorus-Plated Mold with Magnetic Compound Fluid Slurry. Materials and Manufacturing Processes 29(11-12):1502-1509. https://doi.org/10.1080/10426914.2014.930953

13. Barman, A., \& Das, M. (2018) Nano-finishing of bio-titanium alloy to generate different surface morphologies by changing magnetorheological polishing fluid compositions. Precision Engineering 51:145-152. https://doi.org/10.1016/j.precisioneng.2017.08.003

14. Wang, Y., Wu, Y., \& Nomura, M. (2017) Fundamental investigation on nano-precision surface finishing of electroless NiP-plated STAVAX steel using magnetic compound fluid slurry. Precision Engineering 48:32-44. https://doi.org/10.1016/j.precisioneng.2016.11.003

15. Shimada, K., Akagami, Y., Fujita, T., Miyazaki, T., Kamiyama, S., \& Shibayama, A. (2002) Characteristics of magnetic compound fluid (MCF) in a rotating rheometer. Journal of Magnetism and Magnetic Materials 252:235-237. https://doi.org/10.1016/S0304-8853(02)00646-7

16. Guo, H., Wu, Y., Lu, D., Fujimoto, M., \& Nomura, M. (2014a) Effects of pressure and shear stress on material removal rate in ultra-fine polishing of optical glass with magnetic compound fluid slurry. Journal of Materials Processing Technology 214(11):2759-2769. https://doi.org/10.1016/j.jmatprotec.2014.06.014

17. Pan, J., Guo, M., Yan, Q., Zheng, K., \& Xiao, X. (2019) Research on material removal model and processing parameters of cluster magnetorheological finishing with dynamic magnetic fields. The International Journal of Advanced Manufacturing Technology 100(9):2283-2297. https://doi.org/10.1007/s00170-018-2747-6

18. Mosavat, M., \& Rahimi, A. (2019) Simulation and experimental study on the effect of abrasive size, rotational speed, and machining gap during ultra-precision polishing of monocrystalline silicon. Colloids and Surfaces A: Physicochemical and Engineering Aspects 57:50-56. https://doi.org/10.1016/j.colsurfa.2019.05.005

19. Fan, Z., Tian, Y., Liu, Z., Shi, C., \& Zhao, Y. (2019) Investigation of a novel finishing tool in magnetic field assisted finishing for titanium alloy Ti-6Al-4V. Journal of Manufacturing Processes 43:74-82. https://doi.org/10.1016/j.jmapro.2019.05.007

20. Kala, P., Kumar, S., \& Pandey, P. M. (2013) Polishing of Copper Alloy Using Double Disk Ultrasonic Assisted Magnetic $\begin{array}{lllll}\text { Abrasive } \quad \text { Polishing. } & \text { Materials } & \text { 28(2):200-206. }\end{array}$ https://doi.org/10.1080/10426914.2012.746704

21. Kala, P., Sharma, V., \& Pandey, P. M. (2017) Surface roughness modelling for Double Disk Magnetic Abrasive Finishing process. Journal of Manufacturing Processes 25:37-48. https://doi.org/10.1016/i.jmapro.2016.10.007

22. Khan, D. A., \& Jha, S. (2018) Synthesis of polishing fluid and novel approach for nanofinishing of copper using ball-end magnetorheological finishing process. Materials and Manufacturing Processes 33(11):1150-1159. https://doi.org/10.1080/10426914.2017.1328112 
23. More, A. K., \& Chanmanwar, R. M. (2019) Experimental investigation of magnetorheological finishing on copper alloy. Materials Today: Proceedings 19:312-316. https://doi.org/10.1016/j.matpr.2019.07.215

24. Zhang, J., Hu, J., Wang, H., Kumar, A. S., \& Chaudhari, A. (2018) A novel magnetically driven polishing technique for internal surface finishing. Precision Engineering 54:222-232. https://doi.org/10.1016/j.precisioneng.2018.05.015

25. Mori, T., Hirota, K., \& Kawashima, Y. (2003) Clarification of magnetic abrasive finishing mechanism. Journal of Materials Processing Technology 143-144:682-686. https://doi.org/10.1016/S0924-0136(03)00410-2

26. Jain, V. K. (2008) ABRASIVE-BASED NANO-FINISHING TECHNIQUES: AN OVERVIEW. Machining Science and Technology 12(3):257-294. https://doi.org/10.1080/10910340802278133

27. Coello, C. A. C., Pulido, G. T., \& Lechuga, M. S. (2004) Handling Multiple Objectives with Particle Swarm Optimization. IEEE Transactions on Evolutionary Computation 8(3):256-279. https://doi.org/10.1109/TEVC.2004.826067

28. Huang, J., Zhang, J. Q., \& Liu, J. N. (2005) EFFECT OF MAGNETIC FIELD ON PROPERTIES OF MR FLUIDS. International Journal of Modern Physics B 19(01n03):597-601. https://doi.org/10.1142/S0217979205029122 
Appendix A Non-dominated optimal solutions recorded in REP

\begin{tabular}{|c|c|c|c|c|c|c|}
\hline NO. & $\mathrm{W}(\mu \mathrm{m})$ & $\mathrm{h}(\mathrm{mm})$ & $\mathrm{n}(\mathrm{rpm})$ & $\mathrm{t}(\min )$ & $\mathrm{Ra}(\mu \mathrm{m})$ & MRA (g) \\
\hline 1 & 3.4 & 1 & 1000 & 150 & 0.0304 & 0.2753 \\
\hline 2 & 3.4 & 1 & 860 & 131 & 0.0314 & 0.2546 \\
\hline 3 & 3.4 & 1 & 640 & 150 & 0.0316 & 0.2527 \\
\hline 4 & 3.4 & 1 & 810 & 131 & 0.0322 & 0.2306 \\
\hline 5 & 3.4 & 1 & 710 & 120 & 0.0329 & 0.2292 \\
\hline 6 & 3.4 & 1.1 & 830 & 123 & 0.033 & 0.2283 \\
\hline 7 & 3.4 & 1.1 & 700 & 111 & 0.0339 & 0.2248 \\
\hline 8 & 3.4 & 1 & 720 & 110 & 0.034 & 0.2198 \\
\hline 9 & 3.4 & 1.2 & 830 & 125 & 0.0343 & 0.2195 \\
\hline 10 & 3.4 & 1.2 & 540 & 131 & 0.0343 & 0.2263 \\
\hline 11 & 1.6 & 1.1 & 430 & 80 & 0.0359 & 0.2166 \\
\hline 12 & 2.6 & 1.4 & 330 & 97 & 0.0366 & 0.2147 \\
\hline 13 & 1.6 & 1.6 & 200 & 99 & 0.0369 & 0.207 \\
\hline 14 & 3.4 & 1.2 & 780 & 101 & 0.037 & 0.2047 \\
\hline 15 & 1.6 & 1.6 & 300 & 87 & 0.0375 & 0.1669 \\
\hline 16 & 1.6 & 1.7 & 390 & 97 & 0.0381 & 0.1637 \\
\hline 17 & 1.6 & 1.6 & 350 & 80 & 0.0382 & 0.1572 \\
\hline 18 & 1.6 & 1.6 & 360 & 86 & 0.0395 & 0.1437 \\
\hline 19 & 1.6 & 1.7 & 210 & 80 & 0.0395 & 0.1372 \\
\hline 20 & 1.6 & 1.7 & 320 & 79 & 0.0395 & 0.126 \\
\hline 21 & 1.6 & 1.7 & 370 & 84 & 0.0399 & 0.124 \\
\hline 22 & 1.6 & 1.8 & 440 & 84 & 0.0406 & 0.1156 \\
\hline 23 & 1.6 & 1.9 & 330 & 85 & 0.0412 & 0.1129 \\
\hline 24 & 2.6 & 1.7 & 360 & 72 & 0.0424 & 0.1069 \\
\hline 25 & 1.6 & 1.9 & 370 & 85 & 0.0427 & 0.0996 \\
\hline 26 & 1.6 & 1.9 & 320 & 80 & 0.0428 & 0.0956 \\
\hline 27 & 1.6 & 1.9 & 330 & 83 & 0.044 & 0.0901 \\
\hline 28 & 1.6 & 1.9 & 310 & 81 & 0.0456 & 0.0843 \\
\hline 29 & 1.6 & 1.9 & 430 & 76 & 0.0463 & 0.0834 \\
\hline 30 & 1.6 & 2 & 360 & 88 & 0.0469 & 0.0815 \\
\hline 31 & 1.6 & 1.8 & 200 & 64 & 0.0472 & 0.0773 \\
\hline 32 & 1.6 & 2 & 380 & 84 & 0.0476 & 0.0757 \\
\hline 33 & 1.6 & 1.9 & 350 & 75 & 0.0481 & 0.0728 \\
\hline 34 & 1.6 & 1.8 & 210 & 55 & 0.0492 & 0.0712 \\
\hline 35 & 1.6 & 1.9 & 270 & 67 & 0.0503 & 0.0644 \\
\hline 36 & 1.6 & 2 & 310 & 71 & 0.0519 & 0.0589 \\
\hline 37 & 1.6 & 1.9 & 210 & 61 & 0.053 & 0.0556 \\
\hline 38 & 1.6 & 2 & 200 & 64 & 0.0538 & 0.0554 \\
\hline 39 & 1.6 & 2 & 350 & 63 & 0.055 & 0.0517 \\
\hline 40 & 1.6 & 1.9 & 200 & 50 & 0.0566 & 0.0477 \\
\hline 41 & 1.6 & 2 & 300 & 58 & 0.0576 & 0.0464 \\
\hline 42 & 1.6 & 2 & 200 & 56 & 0.0589 & 0.0457 \\
\hline
\end{tabular}




\begin{tabular}{ccccccc}
43 & 1.6 & 1.8 & 200 & 40 & 0.0591 & 0.045 \\
44 & 1.6 & 2 & 200 & 50 & 0.062 & 0.0403 \\
45 & 1.6 & 1.9 & 200 & 37 & 0.0634 & 0.0386 \\
46 & 1.6 & 1.8 & 200 & 30 & 0.066 & 0.0365 \\
47 & 1.6 & 2 & 200 & 44 & 0.0663 & 0.0357 \\
48 & 1.6 & 1.9 & 200 & 30 & 0.0705 & 0.0321 \\
49 & 1.6 & 2 & 200 & 36 & 0.0714 & 0.0312 \\
50 & 1.3 & 2 & 200 & 30 & 0.08076 & 0.0272 \\
\hline
\end{tabular}

